\title{
Self-tuning insulin adjustment algorithm for type 1 diabetic patients based on multi-doses regime
}

doi:10.1533/abbi.2004.0031

D. U. Campos-Delgado ${ }^{1}$, R. Femat ${ }^{2}$, M. Hernández-Ordoñez ${ }^{3}$ and A. Gordillo-Moscoso ${ }^{4}$

${ }^{1}$ Universidad Autónoma de of San Luis Potosí, Facultad de Ciencias, Av. Salvador Nava s/n, Zona Universitaria, C.P. 78290, S.L.P., México

${ }^{2}$ Div. Mat. Aplicadas y Sistemas Computacionales, IPICYT, San Luis Potosí, S.L.P., México

${ }^{3}$ Universidad Autónoma de of San Luis Potosí, CIEP, Facultad de Ingeniería, Mexico

${ }^{4}$ Universidad Autónoma de San Luis Potosí, Facultad de Medicina, San Luis Potosí, S.L.P., México

\begin{abstract}
A self-tuning algorithm is presented for on-line insulin dosage adjustment in type 1 diabetic patients (chronic stage). The algorithm suggested does not need information of the patient insulin-glucose dynamics (model-free). Three doses are programmed daily, where a combination of two types of insulin: rapid/short and intermediate/long acting is injected into the patient through a subcutaneous route. The doses adaptation is performed by reducing the error in the blood glucose level from euglycemics. In this way, a total of five doses are tuned per day: three rapid/short and two intermediate/long, where there is large penalty to avoid hypoglycemic scenarios. Closed-loop simulation results are illustrated using a detailed nonlinear model of the subcutaneous insulin-glucose dynamics in a type 1 diabetic patient with meal intake.
\end{abstract}

Key words: Diabetes, self-tuning algorithm, multi-doses regime, insulin adjustment.

\section{INTRODUCTION}

The diabetes mellitus is a group of metabolic diseases characterized by a high blood glucose concentration (hyperglycemia) resulting from defects in insulin secretion, insulin action, or both phenomena. This complication has largely exceeded its growth expectative, and its impact in the worldwide health-care problem has raised the interest of the scientific community to provide control algorithms that can be implemented for the real-time patient treatment (Carson and Deutsch 1992; Bellazzi et al 2001; Parker et al 2001). In a type 1 diabetes mellitus (TIDM) patient, the pancreas is not able of producing the insulin at all. This problem can produce short- and long-term illnesses (diabetes coma, nephropathy, retinopathy, and other tissue damage) due to the variations in the blood glucose

Corresponding author:

Dr D. U. Campos-Delgado

Universidad Autónoma de of San Luis Potosí

Facultad de Ciencias, Av. Salvador Nava s/n

Zona Universitaria, C.P. 78290, S.L.P., México

Email: ducd@fciencias.uaslp.mx level (BGL). As a matter of fact, long-term complications of diabetes include, among others, peripheral neuropathy with risk of amputation (Lebovitz 1998). As a result, the BGL has to be monitored externally to maintain it regulated by applying insulin infusions in a regular scheme. Meanwhile, in a healthy patient, the insulin released by the pancreas maintains the basal blood glucose concentration around euglycemic (normoglycemic) levels 70$120 \mathrm{mg} / \mathrm{dl}$. Hence, the pancreas provides a basal rate of $\approx 22 \mathrm{mU} / \mathrm{min}$ (Lebovitz 1998; Bode 2004), and it increases this amount during meal intakes (postprandial peak), in order to process the glucose absorbed from the gut. Consequently, in the absence of insulin, the blood glucose level for a TIDM patient can decrease or increase above euglycemic levels (hypoglycemia and hyperglycemia, respectively) for long periods of time. In fact, the TIDM patient requires external insulin for survival. However, the Diabetes Control and Complications Trial (DCCT) (DCCT 1993) showed that an intensive insulin therapy can reduce the incidence of long-term illnesses. Therefore, an intensive therapy is encouraged for TIDM patients prescribed either by a continuous-infusion pump (CIP), or a multiple daily injection regimen (MDIR). On the other hand, it was also noticed in DCCT (1993) that a possible side effect 
Table 1 Insulin characteristics after subcutaneous infusion

\begin{tabular}{lllc}
\hline Type & \multicolumn{3}{c}{ Action (hours) } \\
\cline { 2 - 4 } & Onset & Peak & Duration \\
\hline Rapid & & & \\
Aspart & $0.17-0.33$ & $1-3$ & $3-5$ \\
Lispro & $0.25-0.50$ & $0.25-0.5$ & $3-4$ \\
Short & & & \\
Regular & $0.5-1$ & $2-3$ & $3-6$ \\
Intermediate & & & \\
NPH & $2-4$ & $4-10$ & $10-18$ \\
Lente & $3-4$ & $4-10$ & $16-24$ \\
Long & & & \\
Ultralente & $6-10$ & $8-24$ & $18-30$ \\
Glargine & $1-2$ & $2-20$ & $20-24$ \\
\hline
\end{tabular}

of an intensive therapy is the propensity to hypoglycemic scenarios in the patient. With this consideration, if an intensive therapy is followed by the patient, the presscribed insulin treatment mus be carefully studied by the physician, and it should be constantly updated according with the results achieved. Nevertheless, the benefits of an intensive therapy have been shown in multiple experimental trials for children and adults (Strowig and Raskin 1998; Lalli et al 1999; Weintrob et al 2003). Hence, it is appealing the idea of an automatic advisory system for the patient in order to update each daily dose of insulin continuously (Bellazzi et al 1995; Bellazzi et al 2001; Miyako et al 2004; Bailey and Haddad 2005).

There are two overall approaches for glucose control (Carson and Deutsch 1992), and they depend on the location of the insulin infusions: (a) subcutaneous (Bellazzi et al 2001), and (b) intravenous (Parker et al 2001). For the intravenous approach, a continuous pump is used to deliver a variable insulin infusion rate to the patient, according with a control algorithm that processes the glucose measurements. Several control methodologies have been suggested: $H_{\infty}$ robust control (Kienitz and Yoneyama 1993; Ruiz-Velazquez et al 2004), model predictive control (Parker et al 1999; Lynch and Bequette 2002), and optimal control (Fisher 1991). However, due to the size of mechanical pumps, this approach is now limited to patients under a hospital treatment. Meanwhile, the subcutaneous approach relies on several therapeutic regimens based on combinations of different types of insulin. In general, the insulin can be classified according with its origin: bovine, porcine, and human; and with its action: rapid (Aspart and Lispro), short (Regular), intermediate (NPH and Lente), and long (Ultralente and Glargine) (APhA Special Report 2001; Dickerson 1999). Table 1 illustrates the dynamic characteristics of the different types of human insulin. For some types of insulin, in Berger and Rodbard (1989); Wilinska et al (2005) a mathematical model was proposed to reproduce the assimilation pattern after a subcutaneous injection. The time evolution of Lispro, Regular, NPH,

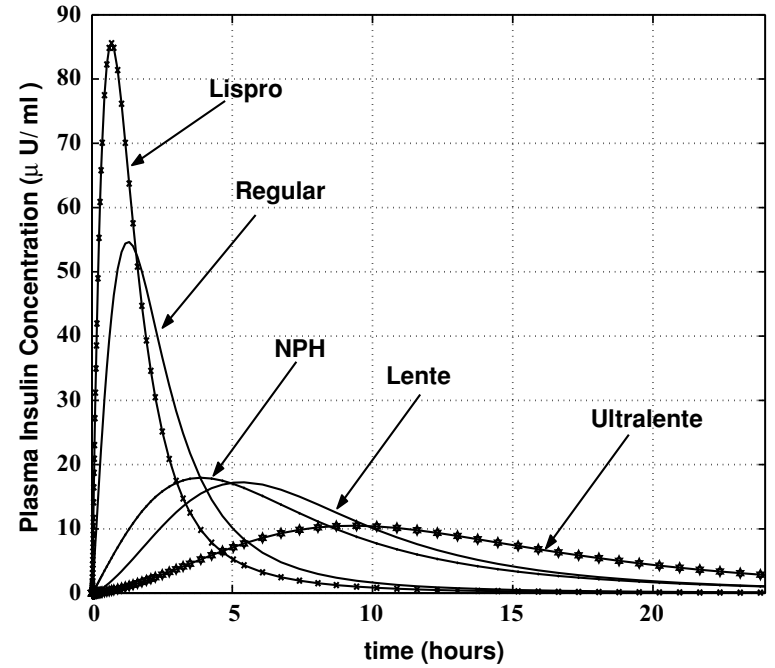

Figure 1 Time evolution of plasma insulin concentration after a subcutaneous insulin infusion of $10 \mathrm{U}$.

Lente, and Ultralente insulins after a $10 \mathrm{U}$ infusion is shown in Figure 1.

The subcutaneous doses are programmed on an MDIR, and they are calculated according with the information gathered by implanted glucose sensors (MiniMed $\left.{ }^{\circledR}\right)$, picks of blood glucose concentration (Accu-Chek ${ }^{\circledR}$ ) or noninvasive blood glucometers (GlucoWatch ${ }^{\circledR}$ ) (Tamada et al 2002), and physician advice. Algorithms for the optimal time and amount of insulin have been suggested in Doyle et al (2001) and Shimauchi et al (1988). Furthermore, in Trajanoski and Wach (1998) a strategy based on neural predictive controllers was adopted. Due to the natural variability of the human metabolism (Lehmann and Deutsch 1998), adaptive control schemes have been proposed in the literature (Carson and Deutsch 1992; Bellazzi et al 2001). In this context, the structure of the minimal model including a peripheral compartment was used in Candas and Radziuk (1994) to perform a parameter estimation to update a control law for glucose regulation. Now, in Bellazzi (2003), an adaptive controller that uses a twolevel architecture is suggested, where a low-level controller uses a fuzzy controller, and an ARX-model for parameters identification and glucose prediction. The high-level module acts as a supervisory system for the low-level controller, and adaptively modifies its characteristics to improve glycemic control. The overall adaptive scheme gives advise to the patient on the insulin protocol (time, type, and amount). On the other hand, in Cavan et al (1998); Hovorka et al (1999) an stochastic model (Bayesian network) was considered, where an algorithm for the optimal insulin dosages is employed based on blood glucose measurements, meal intakes (carbohydrates equivalents), and past insulin injections. This approach (Diabetes Advisory System, DIAS) has been evaluated with good results compared with expert advise of a specialist nurse (Cavan et al 1998). Moreover, using the information of the insulin sensitivity for the patient, the optimum insulin 
dose and timing were calculated by a linear programming algorithm in Shimauchi et al (1988). Also, fuzzy logic has been suggested to incorporate expert knowledge in the disease treatment based on an MDIR (Campos-Delgado et al 2003).

In the overall picture, the blood glucose regulation in TIDM patients presents a challenging interdisciplinary problem that can be approached from different points of view: systems, control, and medicine. However, in order to provide significant advances in the treatment of this disease, the knowledge from these areas must be merged together with a common and clear objective: an effective patient treatment. In this paper, a self-tuning algorithm is suggested to automatically adjust the insulin dosages in an MDIR, using the information of the glucose deviation from euglycemics. The proposed scheme does not need the information of the equivalent carbohydrates by each meal as in Bellazzi (2003); Cavan et al (1998) and Hovorka et al (1999). Moreover, the algorithm does not rely on a direct glucose prediction or estimation to evaluate the insulin adjustments. The paper is organized as follows. The problem statement and performance index are detailed in section "Problem Statement and Control Methodology". Section "MDIR Strategies" defines several MDIR based on combinations of insulin in the formulation, and the self-tuning dosage control structure is detailed in Section "Self-Tuning Insulin Algorithm". Section "Diabetic Patient Modeling" presents briefly the mathematical modeling of the insulin-glucose dynamics in a TIDM patient. Finally, Section "Closed-Loop Simulation Results" introduces the closed-loop simulation results with four testing scenarios and Section "Conclusions" presents concluding remarks and future work.

\section{PROBLEM STATEMENT AND CONTROL METHODOLOGY}

According to Mexican customs, three major meals are taken per day: breakfast (desayuno) (7:00-10:00 hours), lunch (comida) (13:00-15:00 hours), and dinner (cena) (20:0022:00 hours); where the comida meal is the major one of the day. Roughly, there is a time interval of $6 \mathrm{~h}$ among each meal of the day. The approach presented in the paper relies in a three daily injections using rapid/short acting insulin (RSAI) to regulate the postprandial peaks, and intermediate/long acting insulin (ILAI) to provide the basal condition. These doses are programmed $10-15 \mathrm{~min}$ before taking a meal for rapid insulin, and 30-60 min for short-action insulin. Due to the delayed action of the ILAI, the doses for lunch-time is omitted, and only an RSAI is injected.

In order to prevent long-term illnesses (DCCT, 1993), the control objective is defined as to regulate the BGL around a normal level, defined as

$$
\mathrm{NGL}=70-120 \mathrm{mg} / \mathrm{dl}
$$

using three daily doses of a preparation of RSAI and ILAI. Furthermore, it is desired to reach this objective minimizing the amount of insulin by the patient. In this control scheme, several glucose measurements are available daily which could be derived from blood samples, in vivo sensors or noninvasive means (Tamada et al 2002). The control problem posed is very demanding because the doses given by a physician can vary abruptly from patient to patient. Moreover, the insulin-glucose dynamics for a TIDM patient are highly nonlinear and can be modified by different parameters like diet, exercise, etc. (Sorensen 1985; Puckett 1992). Note that a diet for the patient is assigned by the physician according with age and weight; however, in most of the cases, the patient cannot follow tightly the amount of carbohydrates per meal assigned. So, the insulin regime should be robust enough to maintain the BGL regulated despite these issues. Due to the clinical implications, the work in this paper is restricted to TIDM patient in a chronical stage.

According with the glucose measurements, the systemic blood glucose deviation from the NGL can be measured as:

$$
\mathcal{F}=\frac{1}{T} \int_{0}^{T} \phi(t) \mathrm{d} t \approx \frac{1}{N} \sum_{k=1}^{N} T_{s} \cdot \phi\left(\kappa T_{\mathrm{s}}\right)
$$

where $N$ is the number of measurements during the measured interval $T, T_{\mathrm{S}}$ represents the sampling interval in the glucose measurements, and $\phi(t)$ (pointwise deviation from NGL) is defined as

$$
\phi(t)=\left\{\begin{array}{cl}
G(t)-120 \mathrm{mg} / \mathrm{dl} & G(t)>120 \mathrm{mg} / \mathrm{dl} \\
\Gamma \cdot[G(t) & \\
-70 \mathrm{mg} / \mathrm{dl}] & G(t)<70 \mathrm{mg} / \mathrm{dl} \\
0 & 70 \leq G(t) \leq 120 \mathrm{mg} / \mathrm{dl}
\end{array}\right.
$$

In definition (3), $\Gamma>1$ represents a constant that imposes a large penalty for reaching hypoglycemic scenarios. Therefore, (i) $\phi(\cdot)$ is positive in the case of a hyperglycemic (above NGL) condition; (ii) it is negative in the case of a hypoglycemic (below NGL) one; and (iii) it should be close to zero ideally. Thus, equation (2) measures the area outside the NGL during the glucose evolution in a given time interval. However, due to the multi-doses control regime and the absorption process of the subcutaneous insulin infusions, the BGL cannot be completely regulated into the interval $[70,120] \mathrm{mg} / \mathrm{dl}$, and there will be time instants where the BGL lies outside the desired interval. As a result, the doses are increased if the index (2) is positive (hyperglycemia), and reduced if the index is negative (hypoglycemia).

A total of five insulin dosages are then tuned:

(1) $I_{\mathrm{r}}^{\mathrm{b}}$ : breakfast dose of RSAI.

(2) $I_{\mathrm{r}}^{1}$ : lunch dose of RSAI.

(3) $I_{\mathrm{r}}^{\mathrm{d}}$ : dinner dose of RSAI.

(4) $I_{\mathrm{nph}}^{\mathrm{b}}$ : breakfast dose of ILAI.

(5) $I_{\mathrm{nph}}^{\mathrm{d}}:$ dinner dose of ILAI. 
Table 2 Three daily doses control regimens

\begin{tabular}{lll}
\hline Breakfast & Lunch & Diner \\
\hline Lispro+NPH & Lispro & Lispro+NPH \\
Lispro+Lente & Lispro & Lispro+Lente \\
Lispro+Ultralente & Lispro & Lispro+Ultralente \\
Regular+NPH & Regular & Regular+NPH \\
Regular+Ultralente & Regular & Regular+Ultralente \\
\hline
\end{tabular}

by using the information of the performance index in equation (2), the previous insulin dosages, and the characteristic peak and duration time of each insulin type.

\section{MDIR STRATEGIES}

According with the pharmacokinetics and pharmacodynamic effects of each type of insulin, several combinations of RSAI and ILAI can be suggested (APhA Special Report 2001; American Diabetes Association 2002; Dickerson 1999). Consequently, Lispro or Regular insulin are combined with NPH, Lente, or Ultralente insulin. Five therapeutic regimes are illustrated in Table 2 . These regimens are also known as flexible insulin regimens or basal-bolus insulin therapy (Hirsch 1999), because they allow the patient to adjust the timing and amount of insulin in accordance with changes in meal carbohydrate content, euglycemic control, or exercise. Note that the mixing of short-acting (Regular) and lente insulin is not recommended, because the absorption dynamics of the mixture can be seriously delayed (American Diabetes Association 2002). At the time of this study, there was not accurate data and models to identify the absorption dynamics of the (rapid-acting) Aspart and (long-acting) Glargine insulin (see Table 1). Hence, it was not possible to simulate an MDIR that could include these insulins in this study.

Initially, in type 1 diabetic patients, the amount of insulin is calculated based on the patient weight, as $0.3-$ $0.8 \mathrm{U} / \mathrm{kg}$ per day. This amount is continuously updated by the physician in collaboration with the patient in order to reach an euglycemic control, and it could change according with food consumption, exercise, illness, stress, hormonal changes, traveling, and any change of routine (APhA Special Report 2001; American Diabetes Association 2002). Hence, it looks promising and rewarding the idea of an automated insulin adjustment algorithm for diabetic patients. In this paper, a combination of a Lispro and NPH is applied for the RSAI and ILAI. As it was reported in Lalli et al (1999), this combination results in less frequent hypoglycemic scenarios and effective glycemic control. However, the approach could also be fitted to a different combination of RSAI and ILAI.

\section{SELF-TUNING INSULIN ALGORITHM}

The self-tuning algorithm assumes that the patient has a diet and exercise regimens prescribed by a physician
(Bode 2004). Hence, the euglycemic control posterior to the insulin infusions is evaluated to suggest tuning. Therefore, each dose is computed by an evaluation of the performance index (2) based on an optimization criteria:

- The breakfast dose of slow insulin $I_{\text {nph }}^{\mathrm{b}}$ is evaluated by measuring equation (2) during the day until the dinner dosage (dinner), and the dinner dose $I_{\mathrm{nph}}^{\mathrm{d}}$ is evaluated during the night and until breakfast. Thus, if the doses are properly chosen, they should regulate the BGL in an euglycemic level $(\mathcal{F} \approx 0)$.

- The rapid insulin doses $\left(I_{\mathrm{r}}^{\mathrm{b}}, I_{\mathrm{r}}^{\mathrm{l}}, I_{\mathrm{r}}^{\mathrm{d}}\right)$ are evaluated during the posterior time following the infusion, until the next dosage is prescribed.

- If at the time of the infusion, the BGL is above $300 \mathrm{mg} / \mathrm{dl}$ (severe hyperglycemic scenario), then to the next dosage an increase of $30 \%$ of the previous dose is added.

- If at the time of the infusion, the BGL is below $50 \mathrm{mg} / \mathrm{dl}$ (severe hypoglycemic scenario), then to the next dosage a reduction of $30 \%$ of the previous dose is subtracted.

The last two criteria are expert rules included in order to avoid extreme situations of hyperglycemia and hypoglycemia. If the breakfast, lunch, and dinner times are denoted by $t_{\mathrm{b}}, t_{1}$ and $t_{\mathrm{d}}$, then five error measures are used for tuning, assuming for simplicity of the notation of continuous variables:

$$
\begin{aligned}
& \mathcal{F}_{\text {nph }}^{\mathrm{b}}=\frac{1}{t_{\mathrm{d}}-t_{\mathrm{b}}} \int_{t_{\mathrm{b}}}^{t_{\mathrm{d}}} \phi(t) \mathrm{d} t \\
& \mathcal{F}_{\mathrm{nph}}^{\mathrm{d}}=\frac{1}{t_{\mathrm{b}}-t_{\mathrm{d}}} \int_{t_{\mathrm{d}}}^{t_{\mathrm{b}}} \phi(t) \mathrm{d} t \\
& \mathcal{F}_{\mathrm{r}}^{\mathrm{b}}=\frac{1}{t_{1}-t_{\mathrm{b}}} \int_{t_{\mathrm{b}}}^{t_{\mathrm{b}}} \phi(t) \mathrm{d} t \\
& \mathcal{F}_{\mathrm{r}}^{\mathrm{l}}=\frac{1}{t_{\mathrm{d}}-t_{\mathrm{l}}} \int_{t_{\mathrm{i}}}^{t_{\mathrm{d}}} \phi(t) \mathrm{d} t \\
& \mathcal{F}_{\mathrm{r}}^{\mathrm{d}}=\mathcal{F}_{\text {nph }}^{\mathrm{d}}
\end{aligned}
$$

These integral can then approximated by summations of sampled values. The tuning rules are given by equations (9) and (10),

$$
\begin{aligned}
& I_{\mathrm{r}}^{i}(k)=I_{\mathrm{r}}^{i}(k-1)+\alpha \mathcal{F}_{\mathrm{r}}^{i}(k)+\beta\left[\mathcal{F}_{\mathrm{r}}^{i}(k)-\mathcal{F}_{\mathrm{r}}^{i}(k-1)\right] \\
& +\psi \cdot I_{\mathrm{r}}^{i}(k-1) \quad i=\mathrm{b}, 1, \mathrm{~d} \\
& I_{\mathrm{nph}}^{i}(k)=I_{\mathrm{nph}}^{i}(k-1)+\gamma \mathcal{I}_{\mathrm{nph}}^{i}(k)+\delta\left[\mathcal{f}_{\mathrm{nph}}^{i}(k)\right. \\
& \left.-\mathcal{I}_{\mathrm{nph}}^{i}(k-1)\right]+\psi \cdot I_{\mathrm{nph}}^{i}(k-1) \quad i=\mathrm{b}, \mathrm{d} \\
& \psi= \begin{cases}\frac{1}{3} & G^{i}>300 \mathrm{mg} / \mathrm{dl} \\
0 & 50 \leq G^{i} \leq 300 \mathrm{mg} / \mathrm{dl} \\
\frac{-1}{3} & G^{i}<50 \mathrm{mg} / \mathrm{dl}\end{cases}
\end{aligned}
$$

where the index $k$ is referred to the actual evaluation and $k-1$ to the past one, $(\alpha, \gamma)$ are positive constants related to the correction steps for each type of dose, and $(\beta, \delta)$ 


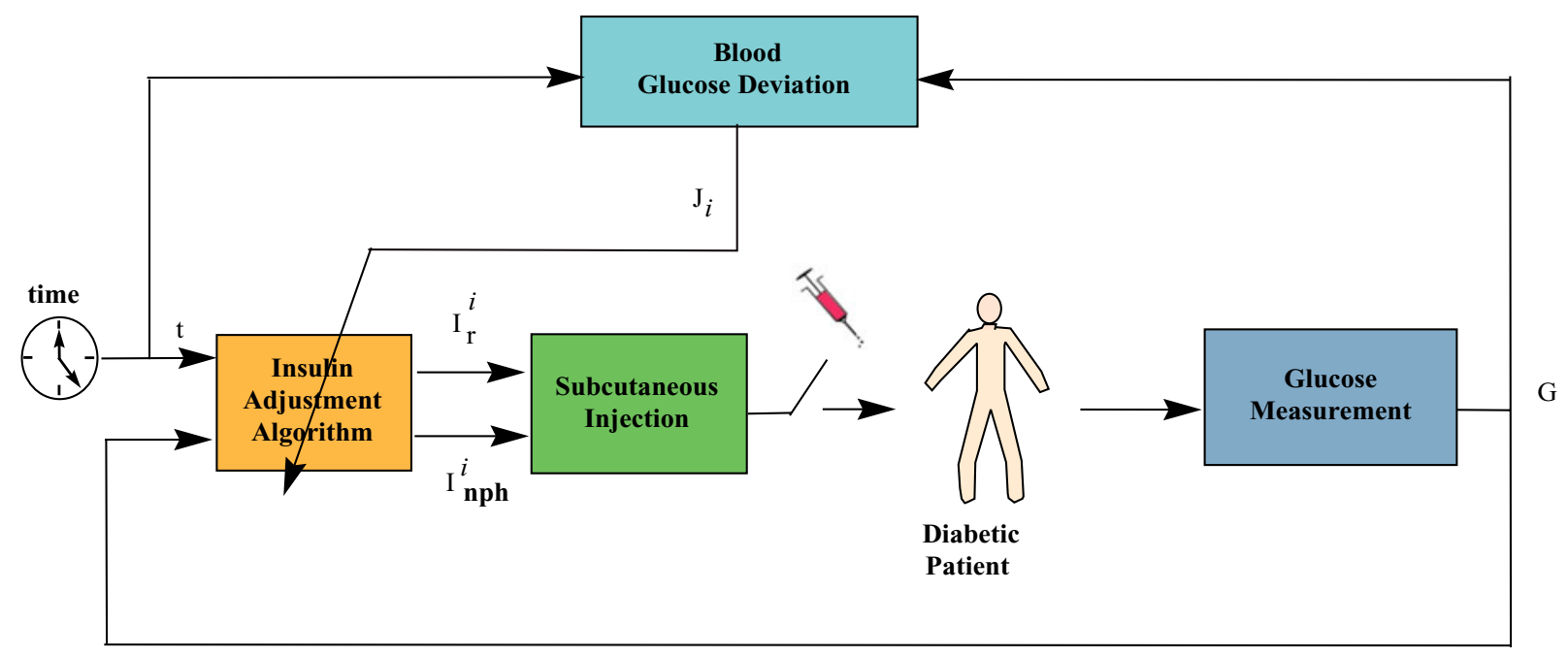

Figure 2 Block diagram of the self-tuning scheme.

are also positive constants that involve a momentum correction that speeds up the convergence to the optimal values (Nocedal and Wright 1999). The glucose measurement $G^{i}=G\left(t_{i}\right) i=\mathrm{b}, 1, \mathrm{~d}$ represents the glucose value at the time of the insulin infusion. The four parameters $(\alpha, \beta, \delta, \gamma)$ can also be time varying, where they are adjusted according with the past history of the performance index. However, this strategy was not pursued in this work, because the simulation showed good performance in the tested scenarios (section "Closed-Loop Simulation Results"). The feedback block diagram of the self-tuning control algorithm is presented in Figure 2. It is important to point out that the tuning rules in equations (9) and (10) can also be visualized as a PID discrete control structure with respect to the glucose deviation that evaluates an error in the euglycemic regulation, plus an expert rule (11) to correct severe hyperglycemic and hypoglycemic scenarios.

\section{DIABETIC PATIENT MODELING}

In this section, the mathematical modeling of a TIDM patient is described. For simplicity, this model is presented in three parts: (1) Insulin-Glucose Compartmental Model: describes the interactions between the different compartments in the body that influence the insulin-glucose dynamics; (2) Glucose Input via Gastric Emptying: presents the modeling of the glucose liberation in the gut following the ingestion of a meal; (3) Subcutaneous Insulin Injection: describes the interactions in the insulin absorption due to subcutaneous injections, and different types of insulin (see Figure 3). The models presented in this section were initially developed by Sorensen (1985); Berger and Rodbard (1989), and Lehmann and Deutsch (1992).

\section{Insulin-glucose compartmental model}

The insulin-glucose model for a type 1 diabetic patient used in this work has a physiological structure based on a compartmental technique (Sorensen 1985; Puckett 1992).

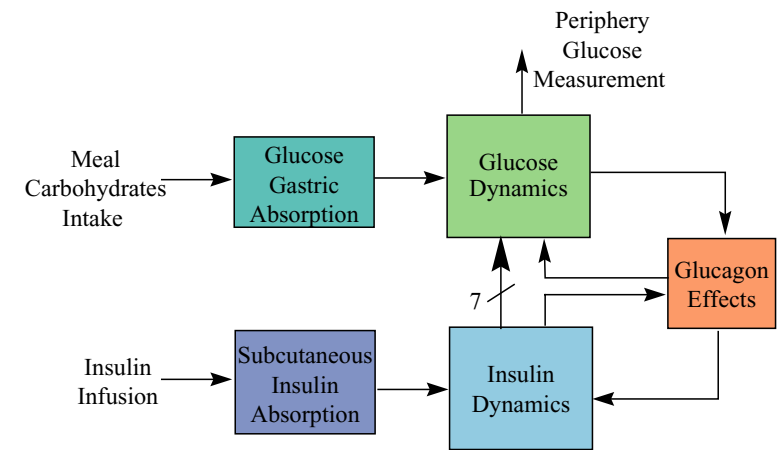

Figure 3 Block diagram of insulin-glucose simulation model.

This model departs from experimental evidence to formulate and validate metabolic processes of the compartmental model on the whole organ and tissue level including counter-regulatory effects. Thus, the insulin-glucose model is governed by 19 nonlinear ordinary differential equations, and is divided into three subsystems:
(1) Glucose,
(2) Insulin, and
(3) Glucagon.

The first two subsystems were modeled for the brain, arterial system (heart/lungs), liver, gut, kidney, and periphery compartments, see Figure 3. The glucagon was modeled as a single blood pool compartment. The system output is the peripheral interstitial glucose, that permits to obtain accurate glucose levels. The system has two inputs:

- Subcutaneous insulin infusion,

- Glucose input via gastric emptying by a meal.

The definition for the dynamic equations of each compartment model are detailed in (Ruiz-Velazquez et al 2004). 
Table 3 Parameters for the absorption process for different types of insulin

\begin{tabular}{llllll}
\hline & Lispro & Regular & NPH & Lente & $\begin{array}{c}\text { Ultra } \\
\text { Lente }\end{array}$ \\
\hline$s$ & 1.80 & 2.0 & 2.0 & 2.4 & 2.5 \\
$a(\mathrm{~h} / \mathrm{U})$ & 0.005 & 0.05 & 0.18 & 0.15 & 0 \\
$b(\mathrm{~h})$ & 1.3 & 1.7 & 4.9 & 6.2 & 13.0 \\
\hline
\end{tabular}

\section{Glucose input via gastric emptying}

The amount of glucose in the gut following the ingestion of a meal containing $\mathrm{Ch}$ milimoles of glucose equivalent carbohydrate is modeled as a first-order differential equation (Lehmann and Deutsch 1992). In this model, the rate of gastric emptying due to a meal is a function of the amount of carbohydrates intake $C h$. This function can have two shapes:

(1) $C h<120 \mathrm{mmol}$ of carbohydrate, then the rate of gastric emptying presents a triangular function, with equally raising and decreasing rates, and a peak value of $120 \mathrm{mmol} / \mathrm{h}$.

(2) $C h \geq 120 \mathrm{mmol}$ of carbohydrate, then a trapezoidal shape is observed, also with equally raising and decreasing rates, but it saturates to $120 \mathrm{mmol} / \mathrm{h}$ during some specific interval.

Finally, the glucose input for a meal intake is given by a proportion of the glucose in the gut.

\section{Subcutaneous insulin injection}

Assume that an insulin dose of $D$ units is injected subcutaneously at time $t_{\text {insulin }}$. Hence, the rate of absorption can be described by

$$
\frac{\mathrm{d} A}{\mathrm{~d} t}=\frac{s\left(t-t_{\text {insulin }}\right)^{s}(a D+b)^{s} D}{\left(t-t_{\text {insulin }}\right)\left[\left(t-t_{\text {insulin }}\right)^{s}+(a D+b)^{s}\right]^{2}}-k_{\mathrm{e}} A
$$

where $k_{\mathrm{e}}$ represents the plasma elimination constant, and the parameters $a, b, s$ are defined in terms of the different types of insulin: Lispro, Regular, NPH, Lente, or Ultralente (Table 3) (Berger and Rodbard 1989). Finally, the plasma insulin concentration due to the subcutaneous injection is proportional to the absorbed insulin. It is assumed that the insulin effect of previous injections is additive (Berger and Rodbard 1989), that is, the insulin plasma concentration depends on the combined effect of the actual and previous dosages. This consideration is not significant for RSAI because its duration is approximately from 3 to $4 \mathrm{~h}$, and the RSAI doses are programmed in periods of $6 \mathrm{~h}$ during the day and $12 \mathrm{~h}$ at night. However, it can be important for ILAI because its duration is from 10 to $18 \mathrm{~h}$.

\section{CLOSED-LOOP SIMULATION RESULTS}

The self-tuning control structure of Figure 2 was simulated using the nonlinear model of the TIDM patient in
Figure 3. The numerical simulation was implemented in MATLAB/Simulink ${ }^{\complement}$. A total of 12 days $(284 \mathrm{~h})$ were simulated with three meals per day:

- Breakfast: 8:00 hours,

- Lunch: 14:00 hours,

- Dinner: 20:00 hours.

Three infusions of insulin are programmed per day by a subcutaneous injection, where a combination of Lispro and NPH insulin is programmed according with the control algorithm for RSAI and ILAI, respectively. Therefore, during the simulation period, a total of 60 doses are computed. The parameters of the tuning algorithm in equations (9) and (10) are shown in Table 4.

In the results of the DCCT (DCCT 1993), the glycosylated hemoglobin $\left(H b A_{1 c}\right)$ test was recognized as a valuable source of information to identify possible risks for diabetic complications. The $H b A_{1 c}$ is a weighted average of blood glucose over a period of 120 days (Bode 2004; Lebovitz 1998), hence it provides an estimation of euglycemic control over the preceding 6-10 weeks. It can be estimated (Rohlfing et al 2002) following the relation

$$
H b A_{1 c}=\frac{\mathrm{MBGL}+77.3 \mathrm{mg} / \mathrm{dl}}{35.6 \mathrm{mg} / \mathrm{dl}}
$$

where MBGL stands for mean blood glucose level during the simulation time. This parameters will be used to evaluate the glycemic control during the simulation tests, note that for a TIDM patient is recommended $H b A_{1 c}<7 \%$ (Bode 2004).

\section{Meals description}

The meals carbohydrate intakes were calculated according with the following profile: male, 30 years old, $80 \mathrm{~kg}$, $1.75 \mathrm{~m}$, number of hours of sleep per day: 7, number of hours of very light activity: 4 , number of hours of light activity: 9 , number of hours of intense activity: 4 , amount of calories per day: $3734 \mathrm{Cal} /$ day. It is considered that $50 \%$ of the calories are coming from carbohydrates, and take that 4 calories are equivalent to $1 \mathrm{~g}$. of carbohydrates $(\mathrm{CH})$. Consequently, it is needed $466.7 \mathrm{~g}$. of carbohydrates per day. Assuming a distribution of this amount of carbohydrates in three meals: $35 \%$ breakfast, $45 \%$ lunch, and $20 \%$ dinner, results in the next meal distribution of carbohydrates:

- Breakfast: $163.34 \mathrm{~g} \mathrm{CH}$,

- Lunch: $210.01 \mathrm{~g} \mathrm{CH}$, and

- Dinner: $93.34 \mathrm{~g} \mathrm{CH}$.

Therefore, the lunch is the heaviest meal of the day according to Mexican customs. During the simulation time (12 days), the amount of carbohydrate intake per meal was varied around the nominal values calculated previously $\pm 10 \%$, but looking to add up to $\approx 3734 \mathrm{Cal} /$ day.

\section{Simulations scenarios}

Four simulation scenarios were tested with the self-tuning adjustment: 
Table 4 Parameters of the tuning algorithm

\begin{tabular}{|c|c|c|c|c|}
\hline \multirow[t]{2}{*}{$\alpha$} & $\beta$ & $\gamma$ & \multirow[t]{2}{*}{$\delta$} & $\Gamma$ \\
\hline & \multicolumn{2}{|c|}{$\mathrm{U} / \mathrm{mg} / \mathrm{dl}$} & & (dimensionless) \\
\hline $7 \times 10^{-5}$ & $7 \times 10^{-5}$ & $4 \times 10^{-5}$ & $5 \times 10^{-5}$ & 5 \\
\hline
\end{tabular}
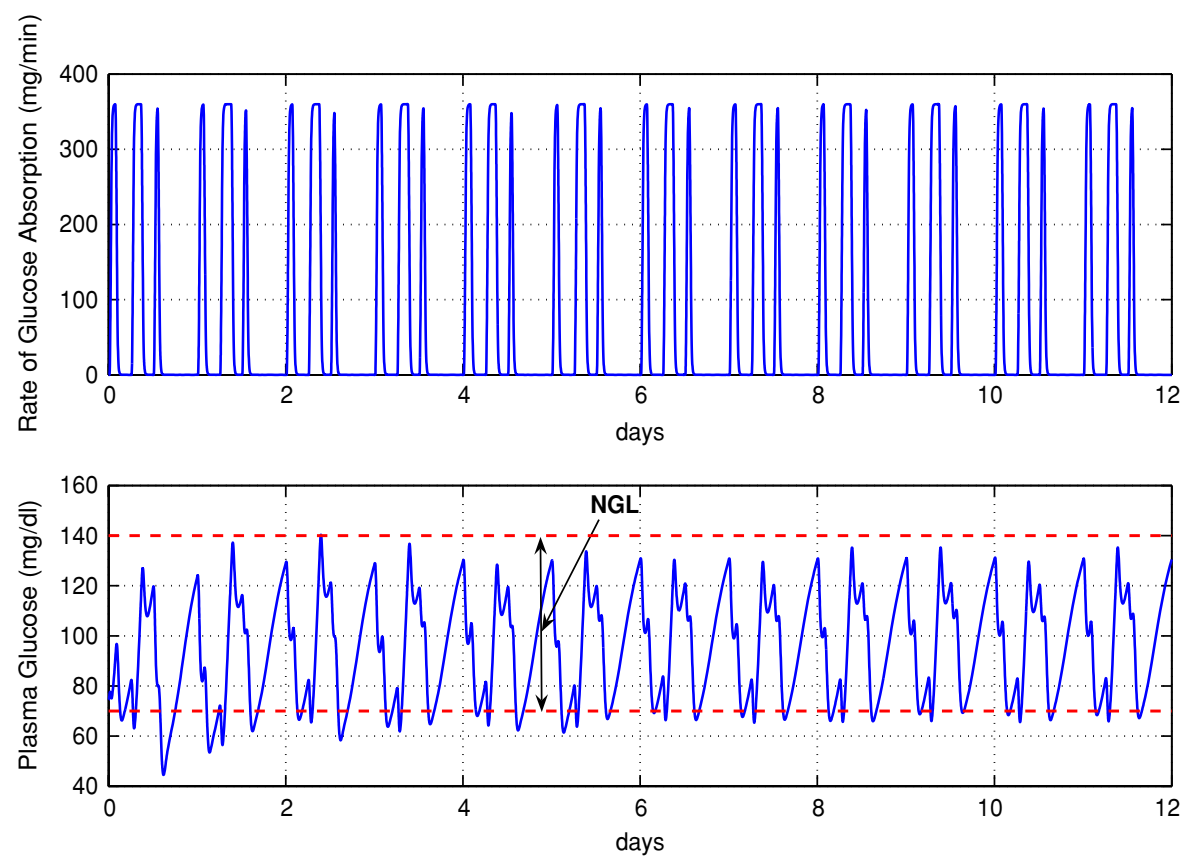

Figure 4 Simulation for Case 1: (top) meal intake, and (bottom) blood glucose level.
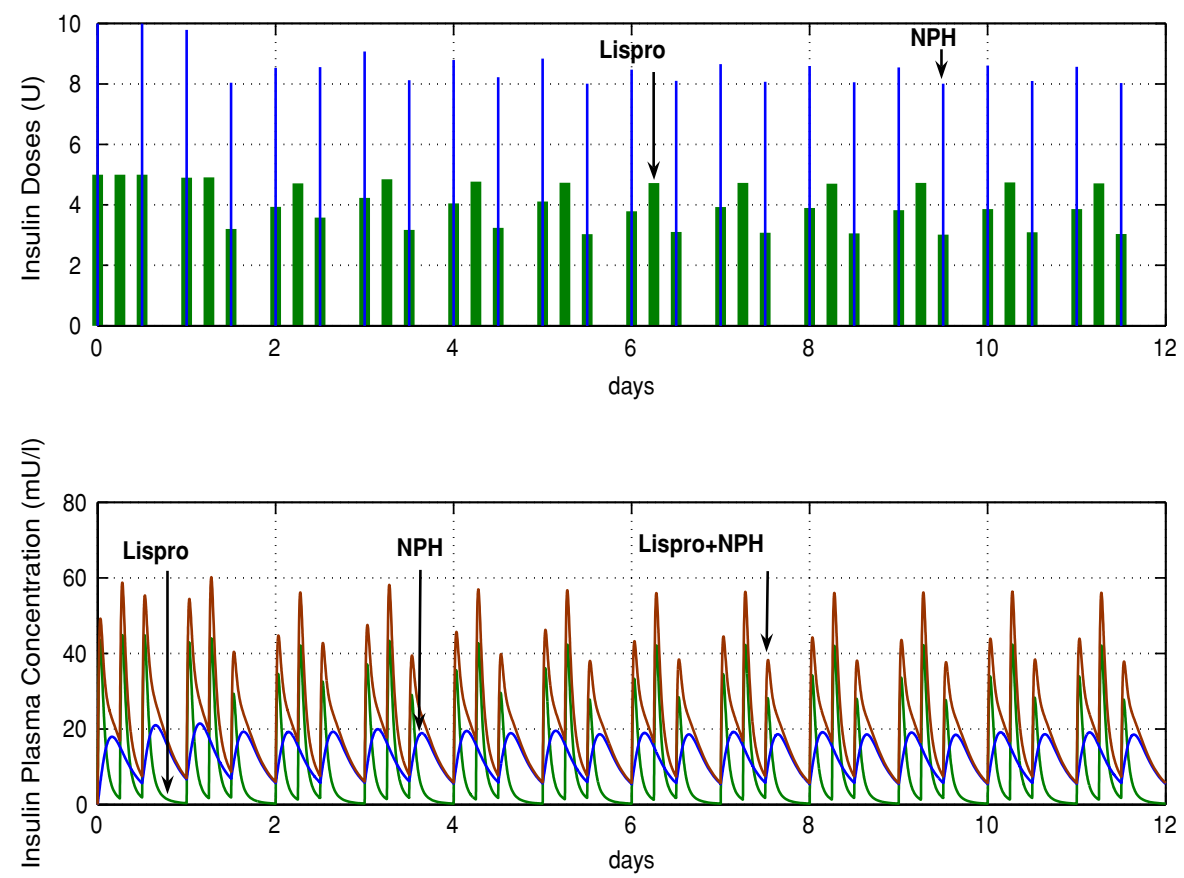

Figure 5 Simulation for Case 1: (top) insulin dosages, and (bottom) insulin plasma concentration. 

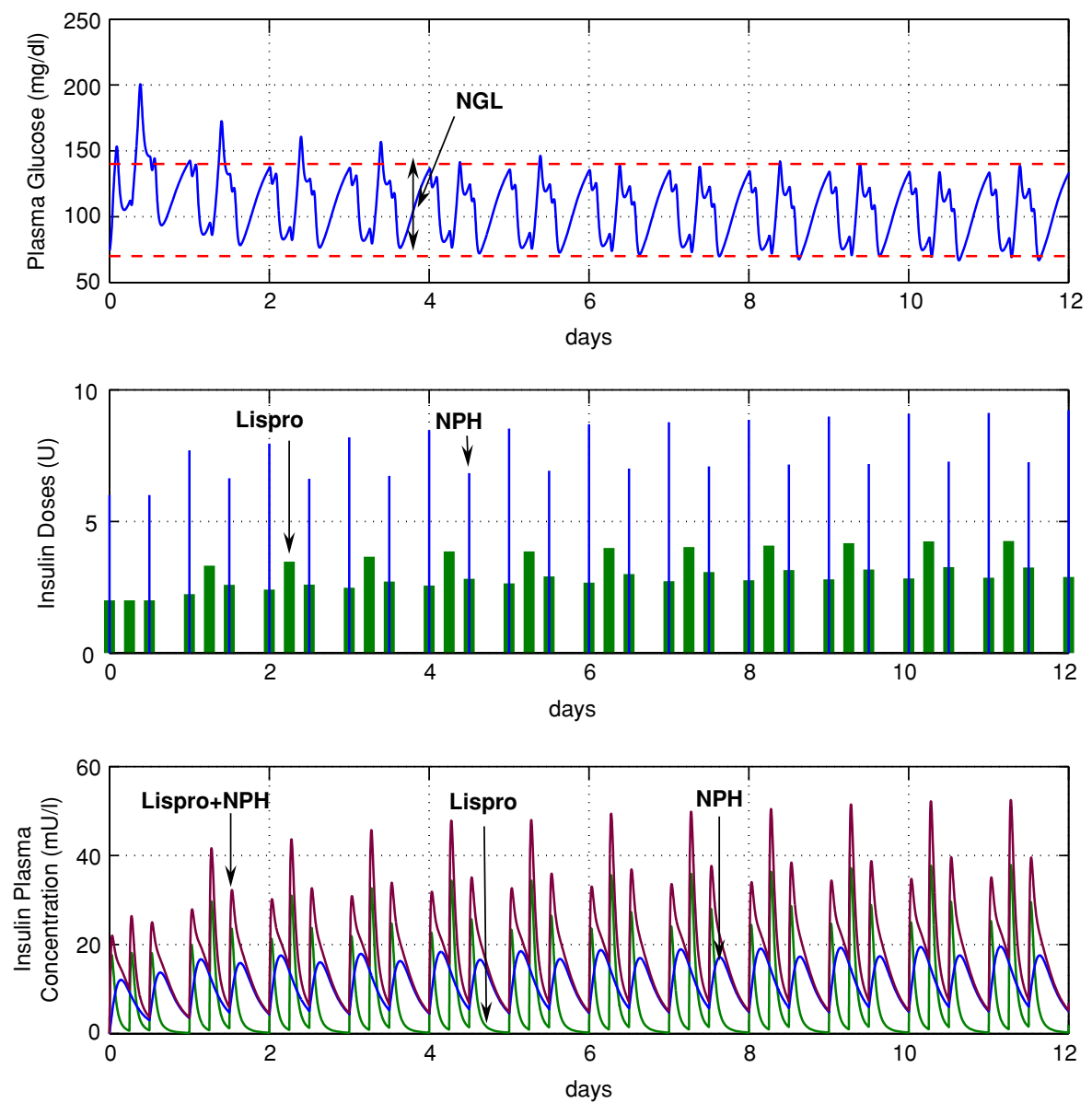

Figure 6 Simulation Case 2: (top) blood glucose level, and (bottom) insulin dosages.
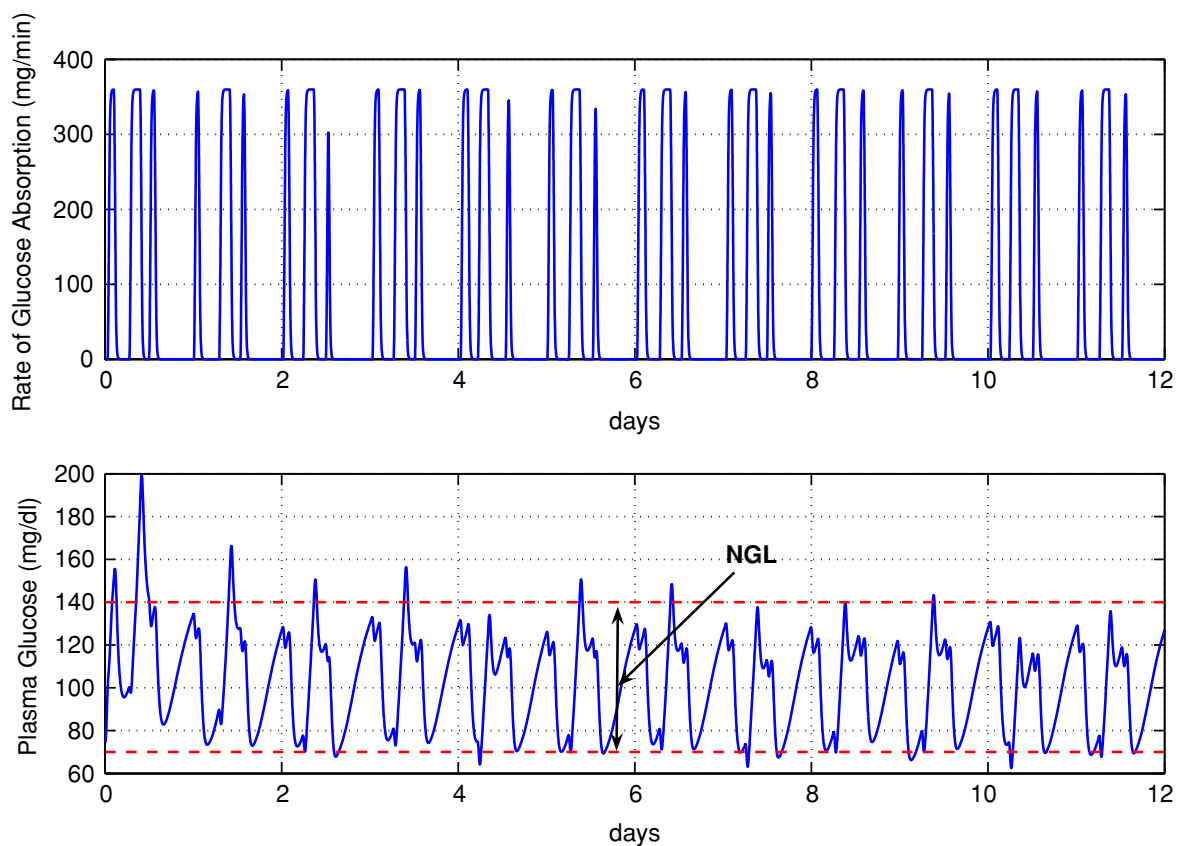

Figure 7 Simulation for Case 3: (top) meal intake, and (bottom) blood glucose level. 

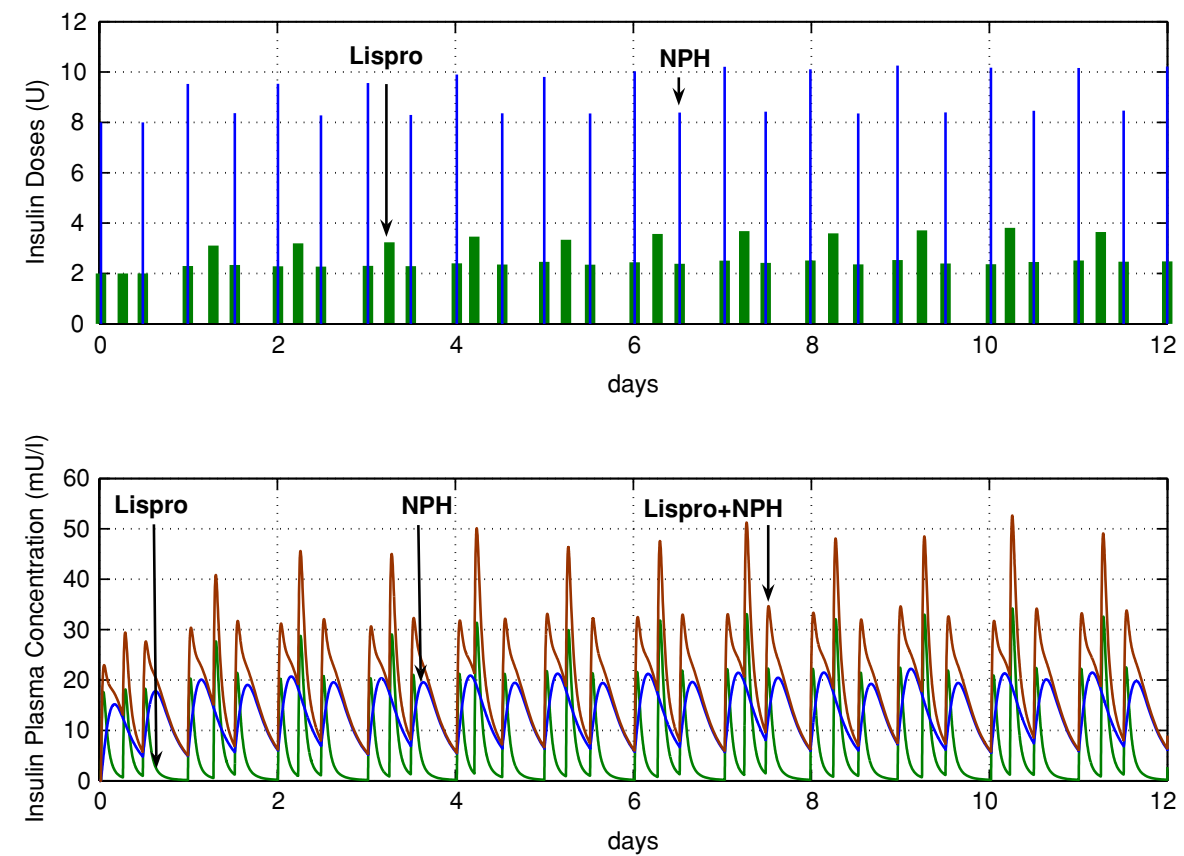

Figure 8 Simulation Case 3: (top) insulin dosages, and (bottom) insulin plasma concentration.
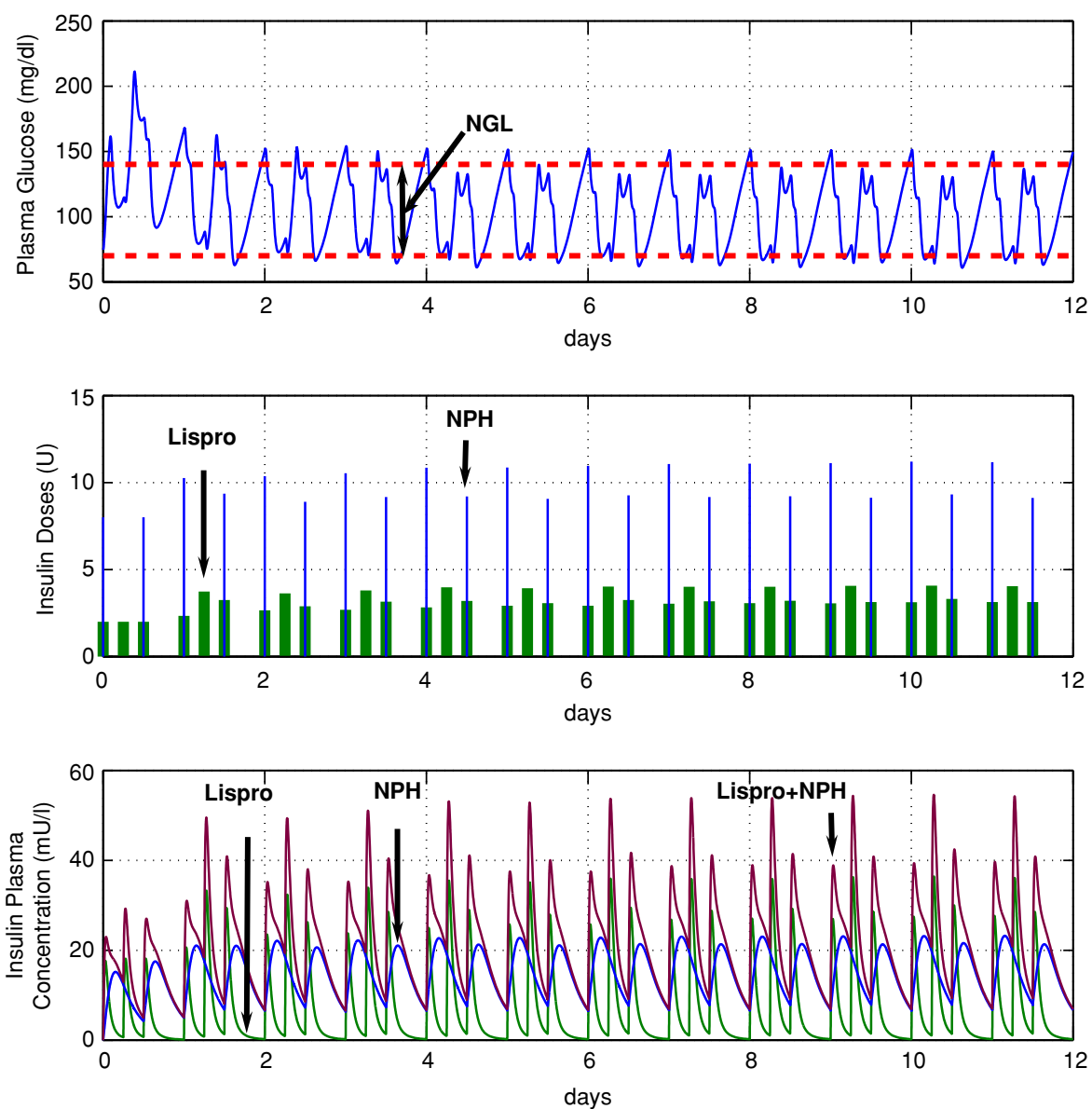

Figure 9 Simulation Case 4: (top) blood glucose level, (middle) insulin dosages, and (bottom) insulin plasma concentration. 
(1) Case 1: the patient starts with high doses for both types of insulin $\left(I_{\mathrm{r}}^{\mathrm{b}}=I_{\mathrm{r}}^{\mathrm{l}}=I_{\mathrm{r}}^{\mathrm{d}}=5 \mathrm{U}\right.$, and $I_{\mathrm{nph}}^{\mathrm{b}}=I_{\mathrm{nph}}^{\mathrm{d}}=$ $10 \mathrm{U})$, as a result the BGL is low initially.

(2) Case 2: consider an opposite scenario to Case 1, the patient starts with small doses for both types of insulin $\left(I_{\mathrm{r}}^{\mathrm{b}}=I_{\mathrm{r}}^{\mathrm{l}}=I_{\mathrm{r}}^{\mathrm{d}}=2 \mathrm{U}\right.$, and $\left.I_{\mathrm{nph}}^{\mathrm{d}}=I_{\mathrm{nph}}^{\mathrm{d}}=6 \mathrm{U}\right)$, producing initially high BGL.

(3) Case 3: the patient varies his carbohydrate intakes during meals by $\pm 25 \%$, and the meal time is also perturbed randomly \pm 30 min using an uniform distribution for both factors. This simulation looks to represent a typical meals distribution for a TIDM patient in chronic condition, because a tight carbohydrates count and time schedule in each meal is difficult for any patient. The patient starts with the following insulin doses $I_{\mathrm{r}}^{\mathrm{b}}=I_{\mathrm{r}}^{\mathrm{l}}=I_{\mathrm{r}}^{\mathrm{d}}=2 \mathrm{U}$, and $I_{\mathrm{nph}}^{\mathrm{b}}=$ $I_{\text {nph }}^{\mathrm{d}}=8 \mathrm{U}$.

(4) Case 4: the insulin-glucose dynamics vary drastically from patient-to-patient. In a previous study (Quiroz et al 2005), it was shown that the parameters related to the hepatic glucose production presented the largest sensitivity in the model response. Thus, in order to analyze this scenario, these parameters were adjusted $10 \%$ at the start of the simulation. The patient starts with the same insulin doses as in Case 3.

In Figures 4 and 5, the results for Case 1 are illustrated. Thus, a regular meal intake is simulated, and due to the initial high dosage values, the BGL is below $70 \mathrm{mg} / \mathrm{dl}$ during long periods of time. However, the algorithms is able to decrease properly the insulin infusions (see Figure 5) in order to regulate the BGL into euglycemics almost constantly. Now, in Figure 6, the opposite scenario is devised. The doses are low and the BGL initially reaches a high value $\approx 200 \mathrm{mg} / \mathrm{dl}$. In this case, the algorithm increases the insulin infusions to reach a regulated BGL. It was observed that the tuning algorithm did not converge to the same insulin doses for Cases 1 and 2, see Figures 5 and 6 .

Finally, in Figures 7-9, the results for Cases 3 and 4 are presented. Through these simulation scenarios the robustness of the tuning algorithm was evaluated. For both cases, the BGL was successfully regulated to euglycemics by the control algorithm. Note that for Case 4 , the insulin infusions need to be raised to compensate the increment by the hepatic glucose production in the insulin-glucose dynamics. Table 5 gives a summary of the simulation results. A hypoglycemic scenario was detected when the BGL dropped below $50 \mathrm{mg} / \mathrm{dl}$. Note that only one hypoglycemic condition was isolated in Case 1, due to the starting high insulin doses, but as shown in Figures 4 and 5 , the control algorithm is capable of promptly adjusting the insulin doses to correct this condition. The calculated glycosylated hemoglobin $H b A_{1 c}$ was always lower to $7 \%$ indicating good euglycemic regulation. Consequently, the results present good robustness in the tuning algorithm proposed in this work.
Table 5 Simulation summary results

\begin{tabular}{lclll}
\hline Case & $\begin{array}{l}\text { MBGL } \\
(\mathrm{mg} / \mathrm{dl})\end{array}$ & $\begin{array}{l}H b A_{1 c} \\
(\%)\end{array}$ & $\begin{array}{l}\text { Hypoglycemic } \\
\text { cases }\end{array}$ & $\begin{array}{l}\text { Minimum } \\
\text { BGL (mg/dl) }\end{array}$ \\
\hline 1 & 99.52 & 4.96 & 1 & 44.49 \\
2 & 113.10 & 5.34 & 0 & - \\
3 & 111.17 & 5.29 & 0 & - \\
4 & 111.20 & 5.29 & 0 & - \\
\hline
\end{tabular}

\section{CONCLUSIONS}

In this paper, a self-tuning algorithm is introduced for insulin dosage adjustment in TIDM patients (chronic stage). Two types of insulin: RSAI and ILAI are considered into the formulation, which are applied in an MDIR through a subcutaneous route. The doses are updated in order to regulate the BGL into euglycemics. The tuning algorithm presents an error correction where a momentum term is used to speed up the convergence. Moreover, the tuning does not need information of the insulin-glucose dynamics (model-free) or meals carbohydrate equivalents, and it is rather simple to compute. Therefore, it has the potential of being implemented in a microcomputer for home treatment. Besides, it can be adapted to another combination of insulin, or another route of application to the patient, like inhaled insulin. The approach and methodology introduced could also be visualized as an alternative for type 2 diabetic patients, and it could be a valuable tool for educational purposes.

\section{ACKNOWLEDGEMENTS}

This research is supported in part by a grant from PROMEP (PTC-28). M. Hernández-Ordoñez acknowledges the financial aid provided by CONACYT through a doctoral scholarship.

\section{References}

American Diabetes Association. 2002. Standards of medical care for patients with diabetes mellitus. Diabetes Care, 25 (Suppl 1):533-49.

APhA Special Report. 2001. New approaches to insulin therapy for diabetes, http://www.pharmacist.com/pdf/insulintherapy-sr.pdf. American Pharmaceutical Association.

Bailey JM, Haddad WM. 2005. Drug dosing control in clinical pharmacology. IEEE Control Syst Mag, 25(2):35-51.

Bellazzi R. 2003. Electronic management systems in diabetes mellitus: Impact on patient outcomes. Dis Manag Health Outcomes, 11(3):159-71.

Bellazzi R, Nucci G, Cobelli G. 2001. The subcutaneous route to insulin-dependent diabetes therapy. IEEE Eng Med Biol, 20:54-64.

Bellazzi R, Siviero C, Stefanelli M et al. 1995. Adaptive controllers for intelligent monitoring. Artif Intell Med, 7:515-40.

Berger M, Rodbard D. 1989. Computer simulation of plasma insulin and glucose dynamics after subcutaneous insulin injection. Diabetes Care, 12:725-36. 
Bode BW. 2004. Medical Management of Type 1 Diabetes, 4th edn. American Diabetes Association, Alexandria, Virginia.

Campos-Delgado DU, Femat R, Ruiz-Velázquez E, et al. 2003. Knowledge-based controllers for blood glucose regulation in type I diabetic patients by subcutaneous route. In: Proceedings of the International Symposium on Intelligent Control, Houston., 3-5 October.

Candas B, Radziuk J. 1994. An adaptive plasma glucose controller based on a nonlinear insulin/glucose model. IEEE Trans Biomed Eng, 41(2):116-24.

Carson ER, Deutsch T. 1992. A spectrum of approaches for controlling diabetes. IEEE Control Syst Mag 12(6):25-31

Cavan DA, Hejlesen OK, Hovorka R et al. 1998. Preliminary experience of the DIAS computer model in providing insulin dose advice to patients with insulin dependent diabetes. Comput Methods Programs Biomed, 56:157-64.

DCCT - The Diabetes Control and Complications Trial Reserach Group. 1993. The effect of intensive treatment of diabetes on the development and progression of long-term complications in insulin-dependent diabetes mellitus. $N \mathrm{Engl} / \mathrm{M}$, 329:977-86.

Dickerson LM. 1999. Insulin therapy in the treatment of diabetes mellitus. http://www.musc.edu/dfm/pharmd/ Med\%20Resident/insulin.pdf.

Doyle FJ III, Srinivasan B, Bonvin D. 2001. Run-to-run control strategy for diabetes management. In: Proceedings of the 23rd Annual EMBS International Conference, Istanbul, Turkey, October 25-28.

Fisher ME. 1991. A semiclosed-loop algorithm for the control of blood glucose levels in diabetics. IEEE Trans Biomed Eng, 38(1):57-61.

Hirsch IB. 1999. Type 1 diabetes mellitus and the use of flexible insulin regimens. Am Fam Phys, 60:2343-56.

Hovorka R, Tudor RS, Southerden D et al. 1999. Dynamic updating in DIAS-NIDDM and DIAS causal probabilistic networks. IEEE Trans Biomed Eng, 46(2):158-68.

Kienitz KH, Yoneyama T. 1993. A robust controller for insulin pumps based on $H_{\infty}$ theory. IEEE Trans Biomed Eng, 40(11):1133-37.

Lalli C, Ciofetta M, Del Sindaco P et al. 1999. Long-term intensive treatment of type 1 diabetes with the short-acting insulin analog lispro in variable combination with $\mathrm{NPH}$ insulin at mealtime. Diabetes Care, 22:468-77.

Lebovitz HE. 1998. Therapy for Diabetes Mellitus and Related Disorders, 3rd edn. American Diabetes Association, Alexandria, Virginia.

Lehmann ED, Deutsch T. 1992. A physiological model of glucose-insulin in type I diabetes mellitus. 7 Biomed Eng, 14:235-42.

Lehmann ED, Deutsch T. 1998. Compartmental models for glycaemic prediction and decision-support in clinical diabetes care: promise and reality. Comput Methods Programs Biomed, 56:193-204.
Lynch SM, Bequette BW. 2002. Model predictive control of blood glucose in type I diabetics using subcutaneous glucose measurements. In: Proceedings of the American Control Conference, Anchorage, AK, May 8-10.

Miyako K, Kuromaru R, Kohno H et al. 2004. Improved diabetes control by using close adjustment algorithms. Pediatr Int, 46:678-84.

Nocedal J, Wright SJ. 1999. Numerical Optimization. Springer-Verlag, New York.

Parker RS, Doyle FJ III, Peppas NA. 1999. A model-based algorithm for blood glucose control in type I diabetes patients. IEEE Trans Biomed Eng, 46(2):148-56.

Parker RS, Doyle FJ III, Peppas NA. 2001. The intravenous route to blood glucose control. IEEE Eng Med Biol, 20:65-73.

Puckett WR. 1992. Dynamic modelling of diabetes mellitus. PhD Dissertation, Chemical Engineering Department, University of Wisconsion-Madison.

Quiroz G. 2005. Dynamic study and geometric properties of the glucose and insulin metabolism in type 1 diabetic patients (in Spanish), Master Thesis. Institute for Scientific and Technological Research in San Luis Potosi (IPICYT), S.L.P., Mexico.

Rohlfing CL, Wiedmeyer H, Little RR et al. 2002. Defining the relationship between plasma glucose and $\mathrm{Hb}_{1 \mathrm{c}}$ Diabetes Care, 25:275-78

Ruiz-Velazquez E, Femat R, Campos-Delgado DU. 2004. Blood glucose control for type I diabetes mellitus: A robust tracking $H_{\infty}$ problem. Control Eng Pract, 12(9):1179-95.

Shimauchi T, Kugai N, Nagata N et al. 1988. Microcomputer-aided insulin dose determination in intesified conventional insulin therapy. IEEE Trans Biomed Eng, 35(2):167-71.

Sorensen JT. 1985. physiologic model of glucose metabolism in man and its use to design and assess improved insulin therapies for diabetes. PhD Dissertation, Chemical Engineering Department, MIT, Cambridge.

Strowig SM, Raskin P. 1998. Improved glycemic control in intensively treated type 1 diabetic patients using blood glucose meters with storage capability and computer-assisted analyses. Diabetes Care, 21:1694-98.

Tamada JA, Lesho M, Tierney MJ. 2002. Keeping watch on glucose. IEEE Spectrum, 39(4):52-7.

Trajanoski Z, Wach P. 1998. Neural predictive controller for insulin delivery using the subcutaneous route. IEEE Trans Biomed Eng, 45(9):1122-34.

Weintrob N, Benzaquen H, Galatzer A et al. 2003. Comparison of continuous subcutaneous insulin infusion and multiple daily injection regimens in children with type 1 diabetes: A randomized open crossover trial. Pediatrics, 112:559-64.

Wilinska ME, Chassin LJ, Schaller HC et al. 2005. Insulin kinetics in type-1 diabetes: Continuous and bolus delivery of rapid acting insulin. IEEE Trans Biomed Eng, 52(1):3-11. 

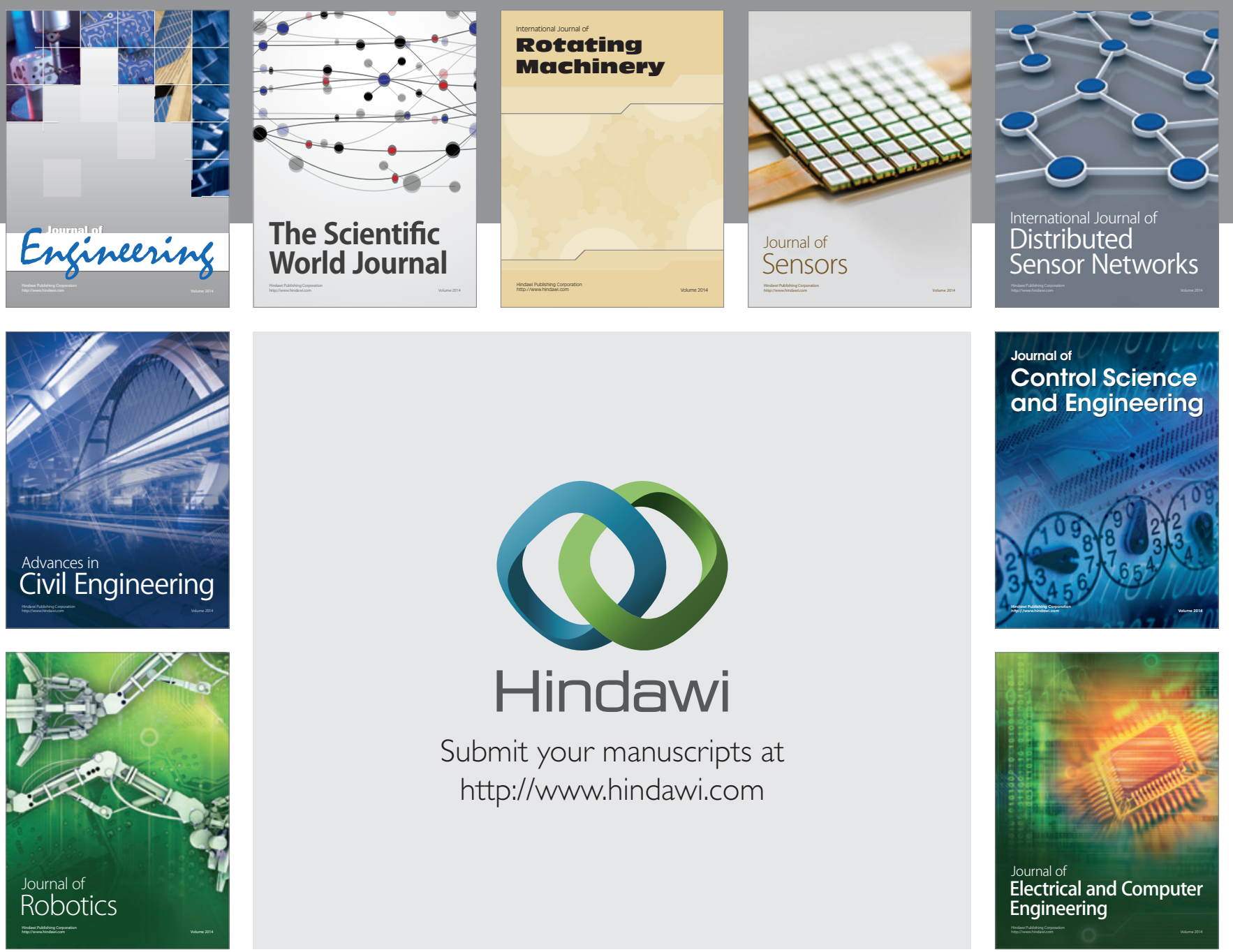

Submit your manuscripts at

http://www.hindawi.com
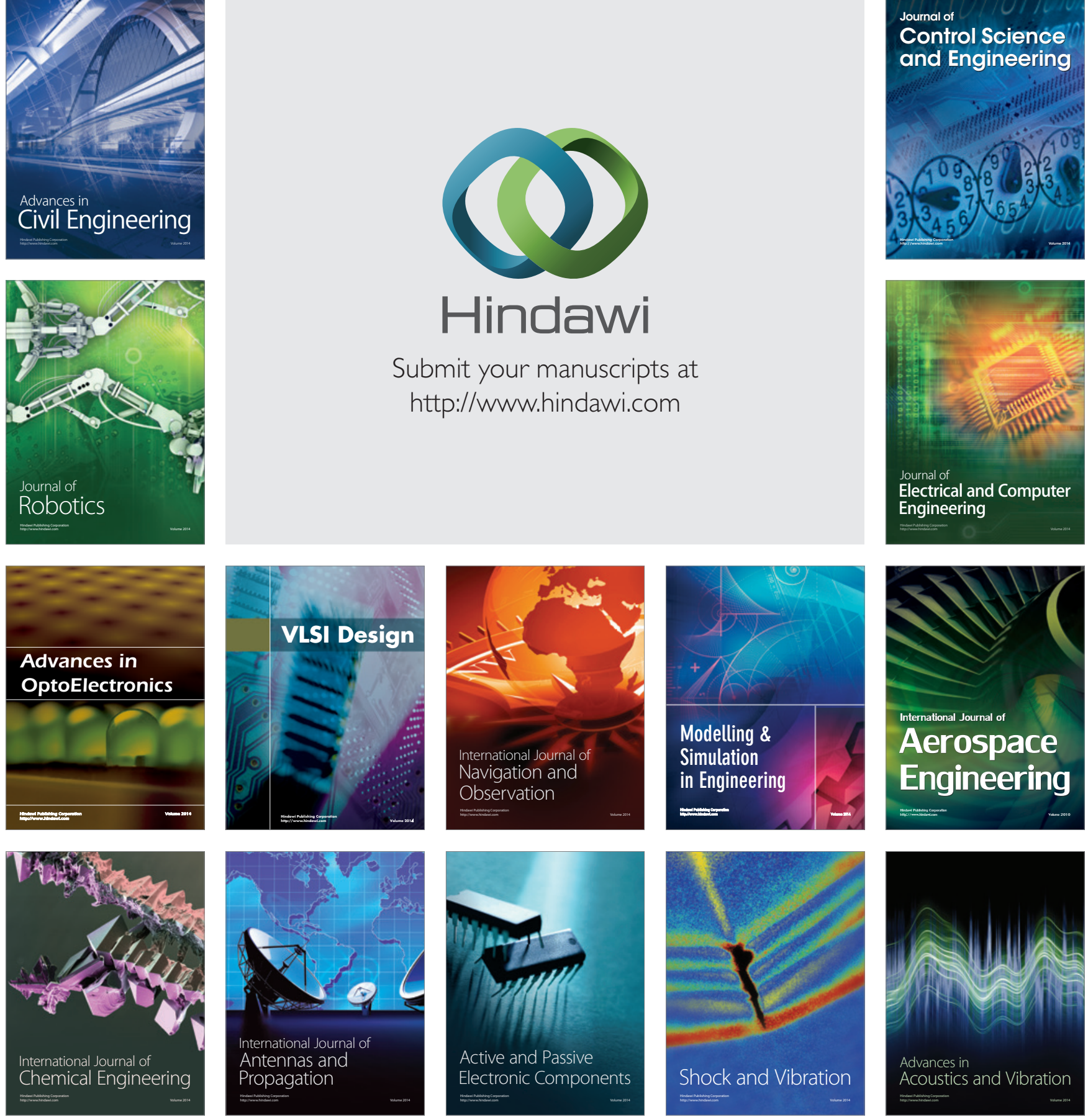